\title{
Fatores determinantes para a escolha da especialidade médica no Brasil
}

\author{
Defining factors for choosing medical \\ specialty in Brazil
}

\author{
José Arnaldo Shiomi da Cruz ${ }^{1,}$ Natascha Silva Sandy, \\ Tiago Ribeiro Vannucchi' ${ }^{1}$ Éder Maxwell Gouveia ${ }^{1}$, Carlo \\ Camargo Passerotti ${ }^{2}$, Homero Bruschini ${ }^{3}$, Miguel Srougi ${ }^{4}$
}

\begin{abstract}
Cruz JAS, Sandy NS, Vannucchi TR, Gouveia EM, Passerotti CC, Bruschini H, Srougi M. Fatores determinantes para a escolha da especialidade médica no Brasil. Rev Med (São Paulo). 2010 jan.-mar.;89(1):32-42.
\end{abstract}

RESUMO: Fundamento. A escolha da especialidade médica é complexa e multifatorial. A importância dos diferentes fatores varia mundialmente e está associada a diferentes valores e sistemas de educação. O objetivo do estudo é identificar os fatores que determinam essa escolha no Brasil. Métodos. Um questionário foi enviado, via internet (eletronic mail), para graduandos dos seis anos do curso de medicina, solicitando informações demográficas e a avaliação de fatores que influenciam na escolha da especialidade médica. Resultados. A idade média foi de 22 anos. Quanto à influência de familiares, $42,5 \%$ dos alunos têm pelo menos um médico como parente próximo, sendo as especialidades mais comuns desse parente: Pediatria, Ginecologia e Obstetrícia e Oftalmologia. A quantidade de respondentes que declararam certeza da carreira a ser seguida ao entrar na faculdade foi $19 \%$, enquanto essa certeza foi de $22,2 \%$ na ocasião da pesquisa. Afinidade pela especialidade e estilo de vida foram fatores avaliados como importantes ou muito importantes por mais de 95\% dos respondentes. Urgência em ganhar dinheiro rápido, tempo curto de residência, alto rendimento inicial e influência familiar foram os fatores avaliados como menos importantes pelos respondentes na escolha de sua especialidade. Quase metade dos respondentes $(49,7 \%)$ descartaram uma especialidade que cogitaram fortemente, as principais razões para isso foram ter descoberto não ter afinidade pela especialidade pela qualidade de vida. Conclusões. Afinidade e estilo de vida são os fatores determinantes da escolha e da rejeição de especialidade médica em nosso meio.

DESCRITORES: Escolha da profissão; Especialidades médicas; Estudo comparativo; Educação médica; Especialização; Educação de pós-graduação em medicina.

Prêmio COMUT 2009 - Área: Medicina Preventiva.

1. Academico(a) da Faculdade de Medicina da Universidade de São Paulo - FMUSP.

2. Médico Assistente da Divisão de Urologia do Hospital das Clínicas da Faculdae de Medicina da Universidade de São Paulo - HCFMUP.

3. Professor Associado da Disciplina de Urologia da Faculdade de Medicina da Universidade de São Paulo - FMUSP.

4. Professor Titular da Disciplina de Urologia da Faculdade de Medicina da Universidade de São Paulo - FMUSP, Chefe da Divisão de Urologia do Hospital das Clínicas da Faculdae de Medicina da Universidade de São Paulo - HCFMUP.

Endereço para correspondência: Rua Corinto, 431 - Apt. 103 - Bloco A - São Paulo - SP, CEP 05586-060 


\section{INTRODUÇÃO}

Mundialmente, observam-se novas tendências na escolha da especialidade médica, com grande declínio na busca por algumas carreiras médicas em contraste ao aumento de interesse por outras. Isso despertou atenção para a pesquisa a respeito do processo de escolha da especialidade médica; processo este caracterizado por grande complexidade e pela influência de múltiplos fatores ${ }^{10,20,31,33,52,55,59,72}$, cada vez mais estudados.

O tema apresentado é de relevância fundamental em educação médica ${ }^{49}$, dada a necessidade de um melhor entendimento desse processo para o desenvolvimento de estratégias que sustentem um equilíbrio dos interesse ${ }^{50}$, promovendo, dessa forma, um equilíbrio da distribuição da força de trabalho médica. Isso se torna importante à medida que as repercussões das novas tendências de alocação de médicos nas especialidades podem se tornar críticas nos próximos anos, especialmente pela falta de profissionais em algumas áreas ${ }^{1}$. Essa questão também tem grande magnitude devido a seu impacto no sistema de saúde pública decorrente do número de profissionais aumentado em determinada área, em detrimento de outras.

A nova distribuição de médicos nas diferentes especialidades e os fatores relacionados a esse novo padrão tem sido muito pouco estudada no Brasil, mas diversos trabalhos americanos ${ }^{7,9,11}$. $12,29,32,36,53,54,56,62$, ingleses ${ }^{23,25,37,38,40,44}$, australianos $28,63,69$ e canadenses ${ }^{49,61,70}$, foram realizados para estudar o assunto.

Dentre os principais fatores relacionados às recentes mudanças na escolha pela especialidade médica, é cada vez mais evidente a valorização do estilo de vida controlável 1,18,24,30,59,60 e o papel de modelos ou mentores (em inglês "role models") 11,19,27,44,48,54,71.

Deve-se observar que a importância dos diferentes fatores varia entre as regiões do mundo, o que possivelmente está associado a diferentes valores e sistemas de educação e também ao próprio sistema de saúde local. Os cinco principais fatores no Canadá ${ }^{70}$, conforme estudo tricêntrico, são, respectivamente: estilo de vida médico, status social, prestígio, status no hospital, e variedade de possibilidades práticas. Na Europa, por sua vez, os determinantes são essencialmente "role models", experiências no internato e disciplinas eletivas ${ }^{19,27,71}$, embora circunstâncias pessoais e gênero também tenham papel importante na escolha da especialidade ${ }^{5,52}$. Nos Estados Unidos, a percepção de estilo de vida controlável parece ser o maior contribuinte para o novo cenário de decisão da carreira médica ${ }^{18}$.

Observa-se que, em países de primeiro mundo, tem havido queda no interesse por medicina da família ${ }^{70}$, medicina interna ${ }^{29}$, prática clínica ${ }^{54}$ e de todos os tipos de prática generalista e de cuidado primário de modo geral ${ }^{12,17,25,37,39,63,70}$; observou-se também queda no interesse por especialidades cirúrgicas ${ }^{7,61}$; aumento do interesse por dermatologia ${ }^{36}$, anestesio$\operatorname{logia}^{36}$, radiologia ${ }^{36}$, medicina de emergência ${ }^{9} e$, de modo geral, também por especialidades cujo estilo de vida pode ser controláve $\left.\right|^{9,60}$. Com relação ao interesse pela medicina acadêmica, não há consenso sobre se está em queda ${ }^{17}$ ou se permanece o mesmo ${ }^{54}$.

Diante dessa nova realidade e da escassez de profissionais em especialidades generalistas, já surgiram tentativas de aumentar o interesse por carreiras neste grupo, como é a "Generalist Physician Initiative" (GPI), criada em 1991, pela fundação Robert Wood Johnson, nos Estados Unidos da América ${ }^{14}$.

Nesse contexto, este trabalho se propõe ao estudo dos fatores determinantes da escolha da especialidade médica entre estudantes de Medicina brasileiros. A pesquisa foca nos fatores que levam à decisão da carreira médica e à rejeição de uma especialidade, não apenas na escolha em si. Nossa realidade é então estudada de maneira comparativa à de países de primeiro mundo, maiores estudiosos do processo de escolha da especialidade médica. Além disso, a pesquisa também teve o objetivo de revisar a literatura médica a respeito do assunto.

\section{MATERIAIS E MÉTODOS}

Um questionário foi enviado, via internet, para os graduandos do primeiro ao sexto ano do curso de Medicina, contendo breves questões demográficas e fatores que podem ter relevância na escolha da especialidade médica, para que tivessem sua importância avaliada (Quadro 1). O questionário foi enviado três vezes para os não respondentes.

Os dados demográficos requeridos foram: idade, ano da faculdade, estado civil, cidade e estado de origem, presença de médico entre familiares próximos e sua especialidade. Os demais itens avaliados, por sua vez, foram selecionados conforme revisão da literatura médica atual e tiveram sua importância medida através da atribuição de uma nota de 1 a 4, sendo: 1 . muito importante; 2 . importante; 3. pouco importante e 4. não importante.

Esses itens foram:

- Estilo de vida médico, qualidade de vida: horas de trabalho, plantões, salários;

- Afinidade com a especialidade, satisfação pessoal/profissional, gratificação;

- Preferência relacionada ao tipo de relação 
Cruz JAS, et al. Fatores determinantes para a escolha da especialidade médica no Brasil.

médico-paciente, preferência por cuidado primário $x$ cuidado hospitalar, preferência por cuidado urgente x não-urgente;

- Exposição à disciplina durante a graduação, contato com a especialidade ao longo do curso;

- Relação com ou conhecimento de um profissional, professor ou orientador que tenha despertado o interesse pela especialidade;

- Influência familiar, parente próximo;

- Tendência/compromisso social;

- Urgência em ganhar dinheiro rápido, tempo curto de residência, alto rendimento inicial;

- Influência de médicos/professores como "modelos".
Foram elaboradas também algumas questões de livre resposta: se o aluno havia entrado na faculdade com a certeza de alguma especialidade e, no caso afirmativo, qual; se o aluno tinha, na ocasião da pesquisa, certeza da carreira que pretendia seguir e, no caso afirmativo, qual; e, finalmente, se o aluno já havia rejeitado alguma carreira que chegou considerar seriamente e, no caso afirmativo, por quê. Essa última pergunta tinha como opções de resposta: qualidade de vida; ter descoberto não ter afinidade pela especialidade; ter se interessado por outra especialidade; ou outro motivo, com espaço para que fosse descrito.

Quadro 1. Pesquisa de escolha da Especialidade Médica

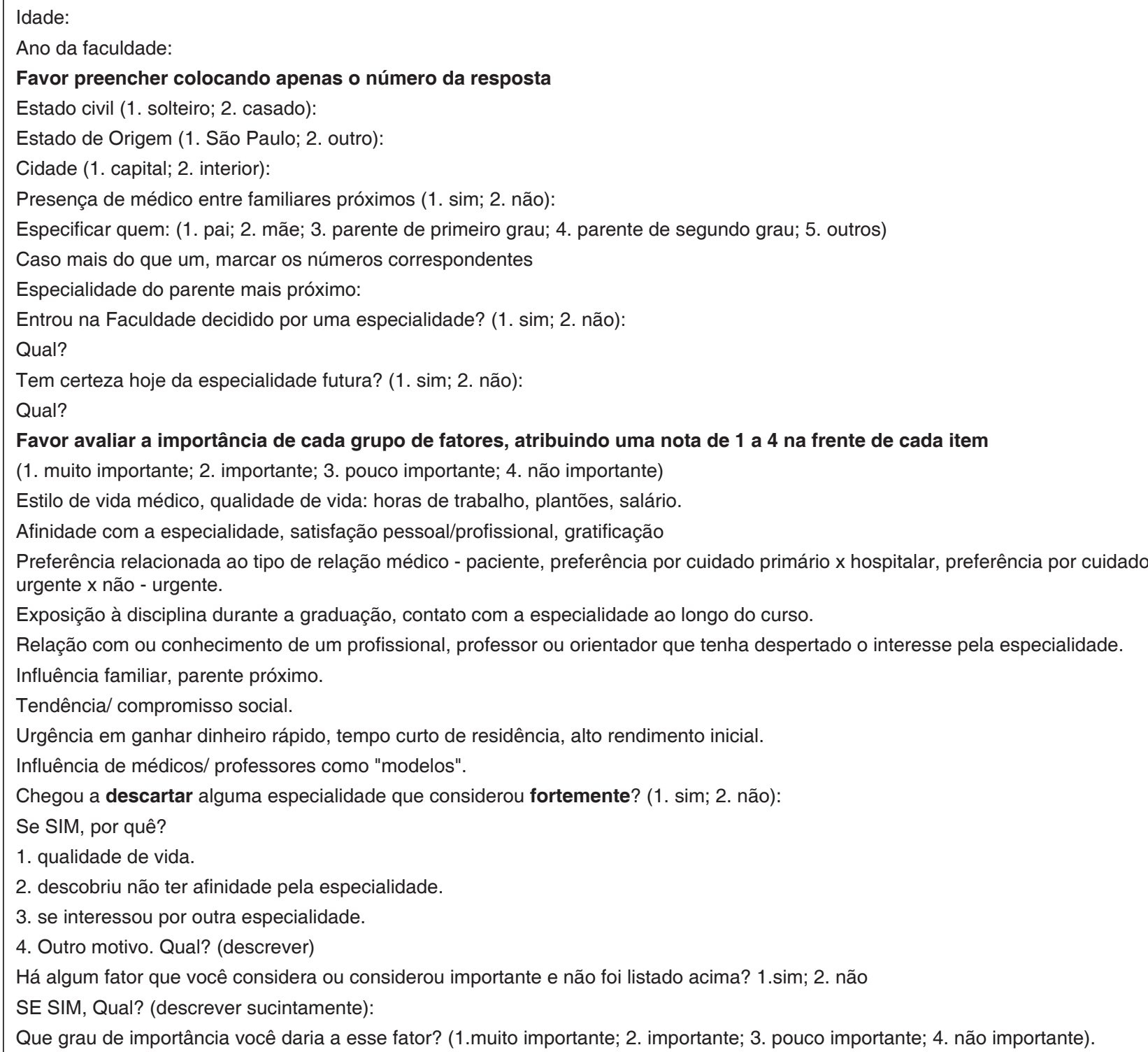


A revisão da literatura médica foi feita através de busca nas bases de dados do "Medline" e "Lilacs", com os termos-chave: "especialidade médica", "escolha da especialidade", "escolha de carreira", "escolha da especialização", "carreira médica", "preferência de carreira" e "preferência de especialidade"; foram selecionados apenas resumos em inglês. A pesquisa no "Medline" forneceu 60 trabalhos a serem avaliados e a pesquisa no Lilacs, apenas cinco.

\section{RESULTADOS}

O índice de resposta obtido foi de 30,9\%, com 325 questionários respondidos de um total de 1,050 (6 turmas de 175 alunos cada). Quarenta por cento das respostas foram obtidas a partir da primeira remessa de e-mails enviados, $28 \%$ a partir da segunda e $32 \%$ a partir da terceira.

A idade dos respondentes variou de 16 a 30 anos, sendo 22 a média e a mediana de idade. Quanto à origem, a maior parte dos alunos referiu ser da capital do estado $(46,20 \%) ; 26,11 \%$ dos respondentes são do estado, mas não da capital; $4,85 \%$ vêm de capitais de outros estados e 5,59\% são do interior de outros estados. A porcentagem de casados entre os respondentes foi de $1,1 \%$.

Um total de $42,5 \%$ dos respondentes afirmaram ter ao menos um médico entre familiares próximos, sendo o pai ou a mãe em $20,6 \%$ e 10,6\% dos casos, respectivamente. A especialidade predominante entre os familiares próximos é Pediatria, seguida por Ginecologia e Obstetrícia e Oftalmologia.

Dos respondentes, $19 \%$ indicaram ter certeza da carreira ao entrar na universidade e 22,2\% indicaram ter certeza, na ocasião da pesquisa, da carreira que pretendiam seguir. No primeiro grupo, as especialidades mais citadas foram: Psiquiatria $(21,6 \%)$, especialidades cirúrgicas, excetuando-se Cirurgia Plástica (13,7\%), Dermatologia (7,8\%), Ortopedia (7,8\%) e Cirurgia Plástica (5,9\%). Todavia, apenas $1 / 3$ desses alunos mantiveram sua posição na ocasião da pesquisa.. Dentre os que afirmaram ter certeza da carreira no momento da pesquisa, as especialidades mais citadas foram: especialidades cirúrgicas (13,6\%), Dermatologia (10,2\%), Cirurgia Plástica $(8,5 \%)$, e Pediatria $(6,8 \%)$. O índice de certeza na ocasião da pesquisa foi significativamente maior no sexto ano $(83,3 \%)$ e oscilou, nos demais anos, entre $2,9 \%$ no segundo e $28,3 \%$ no quarto ano.

Na análise geral dos dados, a importância dos fatores avaliados foi distribuída da seguinte maneira na Tabela 1.

Tabela 1. Pesquisa de escolha da especialidade médica

\begin{tabular}{lcccc}
\hline Fator & $\begin{array}{c}\text { Muito } \\
\text { importante }\end{array}$ & Importante & $\begin{array}{c}\text { Pouco } \\
\text { importante }\end{array}$ & $\begin{array}{c}\text { Não } \\
\text { importante }\end{array}$ \\
\hline Estilo de vida médico & $52,6 \%$ & $42,1 \%$ & $4,5 \%$ & $0,8 \%$ \\
Afinidade com a especialidade & $91 \%$ & $8,3 \%$ & $0,4 \%$ & $0,4 \%$ \\
Preferências relacionadas ao tipo de cuidado & $27,9 \%$ & $54,3 \%$ & $16,6 \%$ & $1,1 \%$ \\
Exposição na graduação/ curso & $22,8 \%$ & $52,4 \%$ & $21,7 \%$ & $3 \%$ \\
Relação com profissional ou professor & $13,6 \%$ & $41,3 \%$ & $36,4 \%$ & $8,7 \%$ \\
Influência familiar & $3,8 \%$ & $13,3 \%$ & $33,7 \%$ & $49,2 \%$ \\
Tendência/compromisso social & $9,1 \%$ & $34,1 \%$ & $38,6 \%$ & $18,2 \%$ \\
Urgência quanto a rendimento, residência curta & $0,4 \%$ & $13,2 \%$ & $47,7 \%$ & $38,7 \%$ \\
$\begin{array}{l}\text { Influência de médicos/ professores como } \\
\text { "modelos" }\end{array}$ & $12,8 \%$ & $39,1 \%$ & $38 \%$ & $10,2 \%$ \\
\hline
\end{tabular}

O grupo de fatores considerado mais importante foi "Afinidade com a especialidade, satisfação pessoal/profissional, gratificação", avaliado como 'muito importante' por $91 \%$ dos alunos entrevistados, e como 'importante' por 8,3\%. Em segundo lugar, ficou o fator "Estilo de vida médico, qualidade de 
Cruz JAS, et al. Fatores determinantes para a escolha da especialidade médica no Brasil.

vida", classificado como 'muito importante' $(52,6 \%)$ ou 'importante' (42,1\%) em 94,7\% dos casos.

Menor importância foi atribuída a "Urgência em ganhar dinheiro rápido, tempo curto de residência, alto rendimento inicial" e "Influência familiar, parente próximo", avaliados como 'pouco importante' ou 'não importante' por $86,4 \%$ e $82,9 \%$ dos respondentes, respectivamente.

Aproximadamente metade dos respondentes $(49,7 \%)$ afirmaram ter descartado uma especialidade que haviam considerado seriamente. Estudando por anos de graduação, observou-se que essa porcentagem foi crescendo progressivamente de 14,3 para $70 \%$ (Figura 1); quanto aos motivos, 63,6\% relataram que descobriram não ter afinidade pela especialidade, e $30,8 \%$ descartaram a especialidade devido à qualidade de vida que ela proporciona.

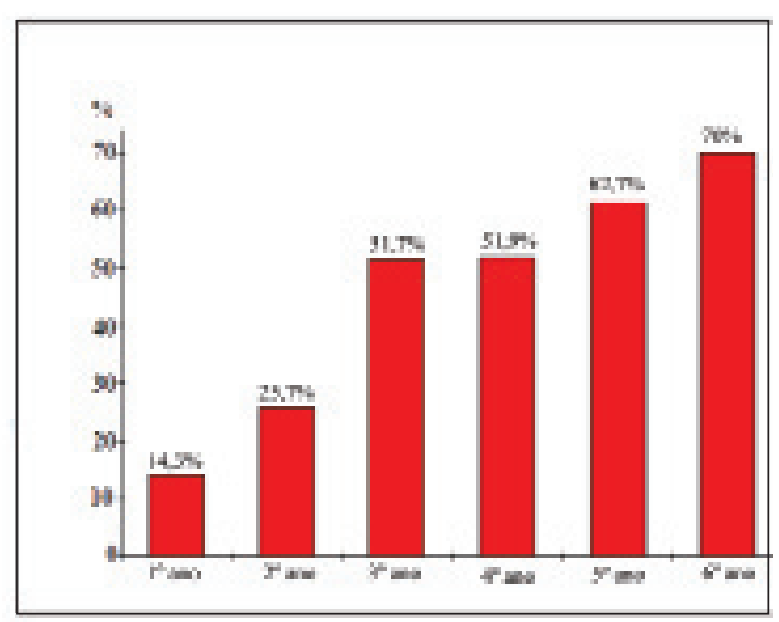

Figura 1. Respondentes que descartaram uma especialidade que foi seriamente considerada

\section{DISCUSSÃO}

A literatura pesquisada contava com poucos estudos longitudinais e multicêntricos, com exceção de um trabalho americano que envolve 11 escolas médicas ${ }^{29}$. A maioria das pesquisas era de estudos transversais e foram realizadas via correio, com índice de resposta variando de $54 \%^{28}$ a $89 \%{ }^{61,70}$. Outros estudos, por sua vez, fazem correlações regressivas, observando dados referentes a uma população de médicos ou estudantes de medicina e ao preenchimento de vagas em programas de residência nos anos correspondentes.

O número de fatores estudados variou, em cada trabalho, de $5^{29}$ a $27^{61} \mathrm{e}$, nas pesquisas mais extensas, foram agrupados em 5 a 7 "clusters" Em geral, a avaliação da importância desses fatores foi realizada com a atribuição de uma nota de 0 a 5 .

No presente estudo, foram avaliados 9 grupos de fatores, nos quais também foram incluídos itens estudados em trabalhos anteriores, a fim de permitir a comparação literária. A avaliação se deu por meio da atribuição de uma nota que variava de 1 a 4, de modo a excluir opção intermediária e, assim, tentar definir um pólo de maior ou menor interesse.

Dentre os diferentes subtemas e abordagens à respeito do processo de escolha da especialidade médica, foram objetivos de pesquisa médica: a influência da escola médica (incluindo origem de fundos para a pesquisa biomédica) ${ }^{11,62}$; o interesse em carreira acadêmica e pesquisa ${ }^{21,53}$; instrumentos psicrométricos para guiar a escolha da carreira médica ${ }^{23}$; a relação médico-paciente nas diferentes especialidades ${ }^{12}$; a estabilidade de uma preferência inicial a uma escolha final ${ }^{61,70,73}$; a influência de características pessoais $3,6,15,16,22,26,35,51$ e demográficas ${ }^{13,28,42,58,64}$; a relação entre fatores e carreiras específicas $2,7,8,9,12,17,25,34,44,47,51,56,61,63,70$; a busca de fatores preditivos ${ }^{32,41,65}$; a rejeição de carreiras inicialmente consideradas ${ }^{37}$; $a$ influência de formação anterior ${ }^{38}$; o desejo de permanecer no país ou praticar no exterior ${ }^{25}$, entre outros.

Houve grande ênfase em algumas questões muito citadas na maioria dos trabalhos ou que, sozinhas, foram objeto de estudos mais aprofundados sobre o tema, tais como: importância do fator financeiro, influência do estilo de vida, influência de professores ou médicos "modelos" (citados em inglês como "role-models"), papel do gênero e da proporção crescente de mulheres em medicina e influência da idade.

Os fatores que influenciam a escolha da especialidade médica são tradicionalmente classificados em intrínsecos e extrínsecos, sendo considerados fatores intrínsecos: idade, sexo, local de nascimento, atributos pessoais e preferências. Fatores extrínsecos, por sua vez, são aqueles relacionados essencialmente ao lar e ao trabalho ${ }^{13,28,58}$, como: circunstâncias familiares, qualidade de vida, flexibilidade, ambiente da prática geral e remuneração. As variáveis demográficas comumente avaliadas são: sexo, idade, etnia, religião, nacionalidade e origem rural.

Muito também se fala sobre a motivação da escolha, definida como o conceito psicológico mais importante em educação e classificada como os fatores, em intrínseca e extrínseca, estando a motivação extrínseca relacionada à obtenção de um resultado específico ${ }^{66}$.

Há grande controvérsia a respeito de qual fator ou grupo de fatores torna-se o maior determinante na decisão da carreira médica. Se por um lado há estudos que identificam os fatores intrínsecos como mais importantes ${ }^{28}$, por outro, também há 
estudos que apontam os fatores extrínsecos como sendo definitivamente determinantes, sobretudo os que dizem respeito a questões econômicas ${ }^{67}$. Entre os trabalhos que definiram do lado dos trabalhos que defendem a importância dos fatores intrínsecos como mais importantes estão artigos que concluíram que a personalidade é o motivo de escolha da carreira médica $3,6,15,16,26,51$; estudos que relacionaram características pessoais e escolha de algumas especialidades específicas (como medicina da família) ${ }^{70}$; estudos que mencionaram uma "personalidade de cirurgião"35; e, ainda, inúmeros estudos que mencionaram as repercussões de fatores como idade e gênero. Enquanto isso há outros trabalhos que concluíram que definitivamente a personalidade não é a influência principal na determinação da carreira. No meio termo, existem também pesquisas que apontaram os fatores intrínsecos como sendo aqueles que influenciam mais fortemente a escolha, mas consideram que eles são também modificados por fatores extrínsecos e experiências pessoais ${ }^{13,58}$. Características pessoais, como estilos de relacionamentos, podem modificar a preferência por carreiras em cuidado primário, em que a relação médico-paciente é de maior intensidade e duração ${ }^{12}$. No presente estudo, observou-se um equilíbrio de importância entre fatores intrínsecos e extrínsecos.

Entre os fatores estudados na literatura, a idade foi principalmente associada à escolha ou rejeição de medicina da família: foi constatado que estudantes mais jovens tendem a rejeitar essa especialidade ${ }^{61}$, enquanto os alunos que a escolhem são, em geral, mais velhos ${ }^{32,70}$. Esse fator se relaciona a outros da seguinte maneira: foi demonstrado que o primeiro grupo tem uma maior porcentagem de estudantes solteiros e influenciados pelo fator "prestigio" 61 , enquanto o segundo grupo se preocupa mais com estilo de vida médico, orientação social e campo variado de prática ${ }^{32,70}$.

É importante observar que a idade média dos estudantes de medicina varia entre os países, bem como a porcentagem de casados, fatores muito provavelmente relacionados. Há, ainda, o relato de que a média de idade dos estudantes de medicina vem aumentando63: nos trabalhos levantados, elas variaram bastante: de $18^{69}$ a $20,45^{2}$ anos, quando se entra na escola médica, e 27,7 anos no último ano de graduação médica em um estudo americano ${ }^{29}$. O fator idade pode explicar parcialmente algumas diferenças observadas entre os fatores considerados importantes nos diferentes países. Neste estudo, foi verificada uma média de idade geral de 22 anos, com médias de 21 anos no primeiro ano de escola médica, e 24 no último (sexto) ano. A porcentagem de casados observada foi $1,1 \%$, muito baixa quando comparada a dos estudos revisados, que chegam a $30 \%{ }^{61}$.

Com relação à influência do gênero, alguns estudos constataram um predomínio masculino na preferência por especialidades cirúrgicas ${ }^{28,61,65}$, contrastando com um predomínio feminino na preferência por prática geral ${ }^{32,40,69}$. Outros fenômenos observados foram que as mulheres têm uma maior preocupação com horas de trabalho razoáveis, têm menos certeza da escolha de carreira e decidem mais tarde que os homens ${ }^{65}$, havendo uma maior rejeição da opção inicial pelo sexo feminino por questões relacionadas à qualidade de vida ${ }^{37}$. Por outro lado, a porcentagem estudantes do sexo feminino que deseja seguir carreira hospitalar se mostrou semelhante à de estudantes do sexo masculino ${ }^{38}$.

A proporção de mulheres entre estudantes de medicina vem crescendo mundialmente ${ }^{63}$, e ainda permanece bastante controversa a questão de como isso tem influenciado as mudanças recentemente observadas nos padrões de escolha da especialidade médica, particularmente se esse crescimento da população feminina seria ou não responsável pela valorização do fator estilo de vida como determinante na escolha da carreira médica. Para alguns autores, a relação entre esses dois fenômenos existe ${ }^{4,63}$ e está associada a uma forte influência, sobre o sexo feminino, da flexibilidade e da compatibilidade com responsabilidades domésticas e familiares ${ }^{63}$. Outros autores, por sua vez, concluíram que fatores relacionados ao estilo de vida são igualmente importantes entre homens e mulheres ${ }^{36,57}$, e, portanto, as mulheres não seriam as maiores responsáveis pela valorização do estilo de vida ${ }^{36}$.

Entre as características da escola médica que podem influenciar a escolha da especialidade pelos alunos, encontram-se: corpo docente, currículo e origem dos fundos de pesquisa. Nos EUA, acreditase que essa influência existe, sobretudo nas escolas com fundos federais para a pesquisa biomédica ${ }^{62}$, havendo uma correlação inversa consistente entre o apoio federal dado à pesquisa biomédica e à porcentagem de alunos que decide por carreiras generalistas ${ }^{11}$. Também há comparações entre a influência do currículo "Problem-Based Learning" (PBL) e a do currículo tradicional ${ }^{17,43,44,46}$. Em nosso meio, ainda existe um grande debate à respeito de qual seria o método mais adequado de ensino em relação tipo de currículo.

Em relação à influência do corpo docente, destaca-se o papel de professores ou "médicos modelos", citados em inglês como "role models", considerados o principal fator na Europa ${ }^{19,27,71}$ e importante fator nos Estados Unidos ${ }^{11}$. Acredita-se que mentores de prática geral tenham influência 
Cruz JAS, et al. Fatores determinantes para a escolha da especialidade médica no Brasil.

positiva mundialmente ${ }^{48}$, e a importância de modelos também é associada a algumas especialidades em particular ${ }^{44,54}$. No presente estudo, a avaliação da "Relação com o conhecimento de um profissional, professor ou orientador que tenha despertado o interesse pela especialidade" e da "Influência de médicos/professores como modelos" foi semelhante, sendo estes fatores considerados muito importantes por apenas $13 \%$ dos respondentes, aproximadamente.

Outro fator de importância emergente é o "estilo de vida controlável", conceito proposto inicialmente em 1989, por Schwartz et al. ${ }^{60}$, que, além de defini-lo ("são as especialidades que permitem ao médico controlar o número de horas dedicadas à prática da especialidade"), ainda classificou nove especialidades nesse grupo (Medicina de Emergência, Radiologia, Oftalmologia, Anestesiologia, Neurologia, Otorrinolaringologia, Patologia, Psiquiatria e Dermatologia) e concluiu que os aspectos relacionados a esse fator são mais influentes na escolha da especialidade médica do que os aspectos relacionados a "motivadores tradicionais" (remuneração, prestígio e duração do treinamento para a especialização). Os critérios de Schwartz foram repetidamente validados ${ }^{18}$ e a importância do conceito introduzido foi ratificada por trabalhos que observaram o estilo de vida como o determinante mais forte das recentes mudanças na escolha de especialidade médica ${ }^{18,24,30,59,60}$. Neste trabalho, o estilo de vida médico foi classificado como o segundo fator mais importante na escolha da especialidade.

Um aspecto importante no processo de especialização é o longo tempo de educação: 3 a 7 anos de residência nos EUA, depois de 4 anos de Escola Médica $^{68 ;} 11$ anos de qualificação no Reino Unido ${ }^{23}$, e uma média que varia, em geral, de 9 e 10 anos no Brasil (6 de escola médica acrescidos de 3 ou 4 anos de residência). Outro aspecto a ser considerado é a dificuldade para trocar de especialidade, mas ainda assim foi relatado que $1 / 3$ dos médicos ingleses acaba trabalhando em outra especialidade que não a escolhida ao final da escola médica ${ }^{23}$.

Algo importante a se investigar é em que momento da formação médica ocorre a escolha da especialidade. Observou-se que, no Brasil, a decisão é feita essencialmente antes do fim da escola médica, quando os médicos recém-formados já devem ingressar no programa de residência - no presente estudo, $83,3 \%$ dos alunos entrevistados do último ano da graduação afirmaram ter certeza da carreira a seguir. Em outros países cujos trabalhos estão em evidência, observa-se uma tendência um tanto diferente: em um estudo australiano, por exemplo, constatou-se que, ao final do terceiro ano após a formatura (PGY3), ainda há, na Austrália, 20\% de médicos não decididos quanto à especialidade médica ${ }^{28}$.

Ainda em relação ao momento de escolha da especialidade médica, foi relatado na literatura que ela tende a ser mais precoce para especialidades cirúrgicas ${ }^{61,68}$, e, como já mencionado anteriormente, é feita mais precocemente pelo sexo masculino ${ }^{65}$. Neste estudo, observamos que $13,7 \%$ dos entrevistados declararam entrar na faculdade decididos por uma especialidade cirúrgica.

É relatado que a preferência inicial por determinada carreira parece estar mais relacionada à escolha final do que se acreditava anteriormente ${ }^{73}$, tendo sido demonstrada associação significativa ${ }^{70}$, principalmente para especialidades cirúrgicas ${ }^{61}$. No presente estudo observou-se que apenas 1/3 dos estudantes que relataram certeza da escolha de uma especialidade ao ingressar na faculdade mantiveram essa decisão, índice válido também para as especialidades cirúrgicas.

Foram observados como fatores que influenciam a mudança após a escolha de carreira ao final da escola médica: quando a carreira foi escolhida, ambiente de escola médica, percepção das especialidades, interesse vocacional e características pessoais; sendo que as principais especialidades deixadas são as de cuidado primário, com motivação relacionada a questões financeiras e de estilo de vida $^{23}$.

Há poucos estudos que avaliaram a rejeição de carreiras inicialmente consideradas, havendo maior foco em fatores positivos da escolha. No Reino Unido, os principais motivadores para que uma especialidade seja desconsiderada foram relacionados à qualidade de vida e aos postos de trabalho ${ }^{37}$. No presente trabalho, observou-se índices crescentes de rejeição de alguma especialidade considerada, do primeiro ao sexto ano, sendo o fator "afinidade" o principal motivo dessa rejeição em todos os anos.

Com relação aos fatores extrínsecos, há pesquisas que mostraram uma valorização cada vez maior da questão financeira, seja sob o aspecto da perspectiva de renda ou sob o aspecto do endividamento de alunos; esse último sendo relacionado até mesmo ao afastamento da área acadêmica ${ }^{21}$. O fato de que a maior parte dos estudos relacionados ao processo de escolha da especialidade médica são realizados por profissionais da área de saúde, com pouco enfoque em questões econômicas, foi alvo de crítica por Thornton e Esposto ${ }^{67}$.

Nos Estados Unidos, existe um instrumento muito importante, o "Annual Medical School Graduation Questionnaire", abreviado como GQ; trata-se de um questionário de graduação cujos dados podem ser 
obtidos para fim de pesquisa ${ }^{21,54}$. Ele é aplicado a nível nacional, sendo administrado para os formandos de todas as escolas médicas dos EUA desde 1978 36,45 pela AMCC (American Association of Medical Collegues). O GQ tem versão on-line desde 2000 e inclui mais de 250 itens, dentre eles, experiências educacionais, dúvidas e planos de carreira. As taxas de resposta deste questionário cresceram

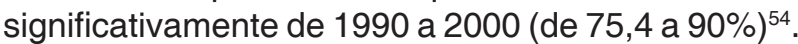
No Brasil, não há nenhum instrumento equivalente ao $\mathrm{GQ}$, mas a instituição de uma ferramenta semelhante poderá gerar um impacto positivo em mudanças e até mesmo incentivo para equalização do sistema.

\section{CONCLUSÕES}

Nesse trabalho, foram identificados os dois principais grupos de fatores que determinam o processo de escolha da especialidade médica no Brasil: afinidade com a especialidade, satisfação pessoal e profissional; e estilo de vida médico e qualidade de vida. Esses são também os principais motivos de rejeição de especialidades previamente consideradas. Observou-se, ainda, que a população de estudantes de medicina pesquisada tem uma média de idade menor do que a encontrada nos países de primeiro mundo, associada a uma menor porcentagem de estudantes casados. Isso pode explicar parcialmente algumas diferenças encontradas na avaliação da importância dos fatores determinantes. Estudos adicionais são necessários para que se possa correlacionar mais fatores com a escolha da especialidade médica.

Cruz JAS, Sandy NS, Vannucchi TR, Gouveia EM, Passerotti CC, Bruschini H, Srougi M. Defining factors for choosing medical specialty in Brazil. Rev Med (São Paulo). 2010 jan.mar.;89(1):32-42.

\begin{abstract}
Background. Choosing medical specialty is complex and depends on multiple factors. The importance of the different factors varies across the world, according to different values and systems of education. The purpose of this study is to identify the determining factors involved in this choice among medical students in Brazil. Methods. A questionnaire was sent by email to medical students from first to sixth year of a selected institution, asking demographic information and the evaluation of factors that may influence the choice of the medical specialty. Results. The mean age was 22 years. Regarding the influence of relatives, $42.5 \%$ of the students has at least one physician as a close relative, the most common specialties of this relative were: Pediatrics, Gynecology and Obstetrics and Ophthalmology. 19\% of the respondents declared being sure of the career when they entered University, while $22.2 \%$ of them were sure about the choice at the occasion of the study. Affinity for the specialty and lifestyle were evaluated as important or very important by more than $95 \%$ of respondents. Urge to earn money fast, short time of medical residency, high initial profit and family influence were the factors evaluated as less important by the respondents. Almost half of the respondents $(49.7 \%)$ rejected a specialty they had strongly cogitated once. The main reasons for this were the lifestyle and the fact that they discovered not having affinity for the specialty. Conclusions. Affinity and medical lifestyle are the defining factors in choosing and rejecting a medical specialty in Brazil.
\end{abstract}

KEY WORDS: Career choice; Comparative study; Education, medical; Specialization; Educational, medical, graduate.

\title{
REFERÊNCIAS
}

1. Azizzadeh A, McCollum $\mathrm{CH}$, Miller CC 3rd, Holliday $\mathrm{KM}$, Shilstone HC, Lucci A Jr. Factors influencing career choice among medical students interested in surgery. Curr Surg. 2003;60:210-3.

2. Baboolal NS, Hutchinson GA. Factors affecting future choice of specialty among first-year medical students of the University of the West Indies, Trinidad. Med Educ. 2007;41(1):50-6.

3. Bartnick LW, Kappelman MM, Berger JH, Sigman B.
The value of the California Psychological Inventory in predicting medical students' career choice. Med Educ. 1985;19:143-7.

4. Barzansky B, Etzel SI. Educational programs in US medical schools, 2000-2001. JAMA. 2001;286:104955

5. Batenburg V, Smal JA, Lodder A, De Melker RA. Are professional attitudes related to gender and medical specialty. Med Educ. 1999;33:489-92. 
Cruz JAS, et al. Fatores determinantes para a escolha da especialidade médica no Brasil.

6. Bland CJ, Meurer LN, Maldonado G. Determinants of primary care specialty choice: a non- statistical metaanalysis of the literature. Acad Med. 1995;70:620-41.

7. Bland $\mathrm{KI}$, Isaacs $\mathrm{G}$. Contemporary trends in student selection of medical specialties: the potential impact on general surgery. Arch Surg. 2002;137(3):259-67.

8. Bobo WV, Nevin R, Greene E, Lacy TJ. The effect of psychiatric third-year rotation setting on academic performance, student attitudes, and specialty choice. Acad Psychiatry. 2009;33(2):105-11.

9. Boyd JS, Clyne B, Reinert SE, Zink BJ. Emergency medicine career choice: a profile of factors and influences from the Association of American Medical Colleges (AAMC) graduation questionnaires. Acad Emerg Med. 2009;16(6):544-9.

10. Burack JH, Irby DM, Carline JD, Ambrozy DM, Ellsbury $\mathrm{KE}$, Stritter FT. A study of medical students' specialty choice pathways: trying on possible selves. Acad Med. 1997;72:534-41.

11. Campos-Oucalt D, Senf J, Watkins AJ, Bastacky S. The effects of medical school curricula, faculty role models, and biomedical research support on choice of generalist physician careers: a review and quality assessment of the literature. Acad Med. 1995;70(7):611-9.

12. Ciechanowski PS, Russo JE, Katon WJ, Walker EA. Attachment theory in health care: the influence of relationship style on medical students' specialty choice. Med Educ. 2004;38(3):262-70.

13. Cohen M. Women in medicine - the Canadian perspective. In: Fourth International Medical Workforce Conference, San Francisco, Nov. 4-7. Sydney: AMWAC; 1999.

14. Colwill JM, Perkoff GT, Blake RL Jr, Paden C, Beachler M. Modifying the culture of medical education: the first three years of the RWJ generalist physician initiative. Acad Med. 1997;72(9):745-53.

15. Coombs RW, Fawzy FI, Daniels ML. Surgeons' personalities: the influence of medical school. Med Educ. 1993;27:337-43.

16. DeForge BR, Sobal J. Investigating whether medical students' intolerance of ambiguity is associated with their specialty selections. Acad Med. 1991;66:49-51.

17. Doman T. Recruitment to academic medicine. Med Educ. 2009;43(4):300-2.

18. Dorsey ER, Jarjoura D, Rutecki GW. Influence of controllable lifestyle on recent trends in specialty choice by US medical students. JAMA. 2003;290:1173-8.

19. Erzurum VZ, Obermeyer RJ, Fecher A, Thyagarajan $\mathrm{P}$, Tan $\mathrm{P}$, Koler AK, et al. What influences medical students' choice of surgical careers. Surgery. 2000;128(2):253-6.

20. Fincher RM, Lewis LA, Rogers LQ. Classification model that predicts medical students' choices of primary care or non-primary care specialties. Acad Med. 1992;67:324-7.

21. Fox M. Medical student indebtedness and the propensity to enter academic medicine. Health Econ. 2003;12(2):101-12.

22. Friedman CP, Slatt LM. New results relating the Myers) Briggs type indicator and medical specialty choice. J Med Educ. 1988;63:325-7.

23. Gale R, Grant J. Sci45: the development of a specialty choice inventory. Med Educ. 2002;36(7):659-66.

24. Gelfand DV, Podnos YD, Wilson SE, Cooke J, Williams RA. Choosing general surgery: insights into career choices of current medical students. Arch Surg. 2002;137:941-7.

25. Goldacre MJ, Davidson JM, Lambert TW. Career choices at the end of the pre-registration year of doctors who qualified in the united kingdom in 1996. Med Educ. 1999;33(12):882-9.

26. Green A, Peters T, Webster D. An assessment of academic performance and personality. Med Educ. 1991;25:343-8.

27. Grifftith 3rd CH, Georgesen JC, Wilson JF. Specialty choices of students who actually have choices: the influence of excellent clinical teachers. Acad Med. 2000;75:278-82.

28. Harris MG, Gavel PH, Young JR, Factors influencing the choice of specialty of Australian medical graduates. Med J Aust. 2005;183(6):295-300.

29. Hauer KE, Durning SJ, Kernan WN, Fagan MJ, Mintz M, O'Sullivan PS, et al. Factors associated with medical students' career choices regarding internal medicine. JAMA. 2008;300(10):1154-64.

30. Jarecky RK, Schwartz RW, Haley JV, Donnelly MB. Stability of medical specialty selection at the University of Kentucky. Acad Med. 1991;66:756-61.

31. Kassebaum DG, Szenas PL. Factors influencing the specialty choices of 1993 medical school graduates. Acad Med. 1994;69:163-70.

32. Kassebaum DG, Szenas PL, Schubert MK. Determinants of the generalist career intentions of 1995 graduating medical students. Acad Med. 1996;71(2):198209.

33. Kiker BF, Zeh M. Relative income expectations, expected malpractice premium costs, and other determinants of physician specialty choice. J Health Soc Behav. 1998;39:152-67.

34. Kolasinski SL, Bass AR, Kane-Wanger GF, Libman BS, Sandorfi N, Utset T. Subspecialty choice: why did you become a rheumatologist? Arthritis Rheum. 2007;57(8):1546-51. 
35. Kozar RA, Anderson KD, Escobar-Chaves SL, Thiel MA, Brundage SI. Preclinical students: who are surgeons? J Surg Res. 2004;119:113-6.

36. Lambert EM, Holmboe ES. The relationship between specialty choice and gender of U.S. medical students, 1990-2003. Acad Med. 2005;80(9):797-802.

37. Lambert TW, Davidson JM, Evans J, Goldacre MJ. Doctors' reasons for rejecting initial choices of specialties as long-term careers. Med Educ. 2003;37(4):312-8.

38. Lambert TW, Goldacre MJ, Davidson JM, Parkhouse J. Graduate status and age at entry to medical school as predictors of doctors' choice of long-term career. Med Educ. 2001;35(5):450-4.

39. Lambert TW, Goldacre MJ, Edwards C, Parkhouse J. Career preferences of doctors who qualified in the United Kingdom in 1993 compared with those of doctors qualifying in 1974, 1977, 1980 and 1983. BMJ. 1996;313:19-24.

40. Lambert TW, Goldacre MJ, Turner G. Career choices of United Kingdom medical graduates of 2002: questionnaire survey. Med Educ. 2006;40(6):514-21.

41. Lawson SR, Hoban JD. Predicting career decisions in primary care medicine: a theoretical analysis. $\mathrm{J}$ Contin Educ Health Prof. 2003;23(2):68-80.

42. Makkai T. Origins and destinations: geographical mobility of medical practitioners from 1966 till 1993. Canberra: Sociology Programme, Research School of Social Sciences, Australian National University; 1995.

43. Martenson DF. Students' approaches to studying in four medical schools. Med Educ. 1986;20:532-4.

44. McParland M, Noble LM, Livingston G, McManus C. The effect of a psychiatric attachment on students' attitudes to and intention to pursue psychiatry as a career. Med Educ. 2003;37(5):447-54.

45. AAMC - Association of American Medical Colleges. Graduation Questionnaire (GQ). Available from: http:// www. aamc.org/data/gq.

46. Mennin SP, Kalishman S, Friedman M, Pathak D, Snyder J. A survey of graduates from the University of New Mexico's conventional and community-orientated problem-based track. Acad Med. 1996;71:1079-89.

47. Meurer LN Influence of medical school curriculum on primary care specialty choice: analysis and synthesis of the literature. Acad Med. 1995;70(5):388-97.

48. Mihalynuk T, Leung G, Fraser J, Bates J, Snadden D. Free choice and career choice: Clerkship electives in medical education. Med Educ. 2006;40:1065-71.

49. Mowbray RM. Research in choice of medical specialty: a review of the literature 1977-87. Aust N Z J Med. 1989;19(4):389-99.
50. Mutha S, Takayama JI, O'Neil EH. Insights into medical students' career choices based on third- and fourthyear students' focus-group discussions. Acad Med. 1997;72(7):635-40

51. Neral SM, Collins J, Gandy MJ, Hampton HL, Morrison JC. Non-cognitive variables and residency choice. J Miss State Med Assoc. 2008;49(11):327-9.

52. Odborg MH, Eriksen TR, Petersson BH. Kønnets betydning for lægerollen. Ugeskr Laeger. 1995;4;157(36):4942-6.

53. Reck SJ, Stratman EJ, Vogel C, Mukesh BN. Assessment of residents' loss of interest in academic careers and identification of correctable factors. Arch Dermatol. 2006;142(7):855-8.

54. Richard GV, Nakamoto DM, Lockwood JH. Medical career choices: traditional and new possibilities. JAMA. 2001;285(17):2249-50.

55. Rosenthal MP, Diamond JJ, Rabinowitz HK, Bauer LC, Jones RL, Kearl GW, et al. Influence of income, hours worked, and loan repayment on medical students' decision to pursue a primary care career. JAMA. 1994;271:914-7.

56. Roubidoux MA, Packer MM, Applegate KE, Aben G. Female medical students' interest in radiology careers. J Am Coll Radiol. 2009;6(4):246-53.

57. Sanfey HA, Saalwachter-Schulman AR, Nyhof-Young JM, Eidelson B, Mann BD. Influences on medical student career choice: gender or generation? Arch Surg. 2006;141:1086-94. [erratum in Arch Surg. 2007;142:197].

58. Schmittdiel J, Grumbach K, Selby JV, Quesenberry CP $J r$. Effect of physician and patient gender concordance on patient satisfaction and preventive care practices. J Gen Intern Med. 2000;15(11):761-9.

59. Schwartz RW, Haley JV, Williams C, Jarecky RK, Strodel WE, Young B, et al. The controllable lifestyle factor and students' attitudes about specialty selection. Acad Med. 1990;65:207-10.

60. Schwartz RW, Jarecky RK, Strodel WE, Haley JV, Young B, Griffen WO. Controllable lifestyle: a new factor in career choice by medical students. Acad Med. 1989;64:606-609. 10 = Scott IM, Matejcek AN, Gowans MC, Wright BJ, Brenneis FR. Choosing a career in surgery: factors that influence Canadian medical students' interest in pursuing a surgical career. Can J Surg. 2008;51(5):371-7.

61. Senf JH, Campos-Outcalt D, Watkins AJ, Bastacky S, Killian C. A systematic analysis of how medical school characteristics relate to graduates' choices of primary care specialties. Acad Med. 1997;72(6):524-33.

62. Shadbolt N, Bunker J. Choosing general practice - a review of career choice determinants. Aust Fam Phys. 
Cruz JAS, et al. Fatores determinantes para a escolha da especialidade médica no Brasil.

2009;38(1-2):53-5.

63. Shapiro MC, Western JS, Anderson DS. Career preferences and career outcomes of Australian medical students. Med Educ. 1988;22:214-21.

64. Soethout MBM, ten Cate TJ, van der Wal G. Factors associated with the nature timing and stability of the specialty career choices of recently graduated doctors in European countries. Med Educ Online [serial online]. 2004;9:24. Available from: http://www.med-ed-online. org

65. Tanaka M, Mizuno K, Fukuda S, Tajima S, Watanabe $Y$. Personality traits associated with intrinsic academic motivation in medical students. Med Educ. 2009;43(4):384-7.

66. Thornton J, Esposto F. How important are economic factors in choice of medical specialty? Health Econ. 2003;12(1):67-73.

67. Torpy JM, Lynm C, Glass RM. JAMA patient page.
Medical specialties. JAMA. 2003;290(9):1268.

68. Ward AM, Kamien M, Lopez DG. Medical career choice and practice location: early factors predicting course completion, career choice and practice location. Med Educ. 2004;38(3):239-48.

69. Wright B, Scott I, Woloschuk W, Brenneis F, Bradley J. Career choice of new medical students at three Canadian universities: family medicine versus specialty medicine. CMAJ. 2004;170(13):1920-4.

70. Xu G, Hojat M, Brigham TP, Veloski JJ. Factors associated with changing levels of interest in primary care during medical school. Acad Med. 1999;74:1011-5.

71. Zeldow PB, Daugherty SR. Personality profiles and specialty choices of students from two medical school classes. Acad Med. 1991;66:283-7.

72. Zeldow PB, Preston RC, Daugherty SR. The decision to enter a medical specialty: timing and stability. Med Educ. 1992;26(4):327-32. 